International Journal of Child, Youth and Family Studies (2012) 2 \& 3: 284-299

\title{
INVESTIGATING THE MULTIMODALITY OF CHILDREN AND YOUTH
}

\author{
Lynda Phillips and Catherine Ann Cameron
}

\begin{abstract}
Capturing lived childhoods without decontextualizing their meaning and still providing information needed by policy-makers and practitioners is a pressing challenge for contemporary researchers. In this paper we provide information to open up such a dialogue via a range of tools we have utilized when investigating well-being. We interrogate bio-socio-ecological approaches to human development to provide relatively holistic pictures of the lived experience of childhood. We utilize various methodologies within this approach to determine what they transactionally facilitate at each level. At the bio-psychological level, for example, controlled, psychologically valid, psychosocial stress procedures expose hormonal responses, yielding valuable information about individual differences in physiological stress reactivity. At the level of the psychological self within a social ecology, we systematically observe children and youth in naturalistic, environmental transactions with the aid of visual methodologies such as Day in the Life filming, and invite the children and their parents and youth to share their reflections on their lived context via focused discussions and interviews. In this paper we discuss new ways of integrating research findings by suggesting Sameroff's (2010) unified theory as an interpretive framework for research within the field of child and youth care.
\end{abstract}

Keywords: children and youth, Day in the Life, bio-socio-ecological methodologies, transactional models

Lynda Phillips, Ph.D. is an Instructor in the Department of Child and Youth Care Counselling at Douglas College, and an Adjunct Professor in the Department of Educational Psychology at Vancouver Island University. E-mail: phillipsly@douglascollege.ca

Catherine Ann Cameron, Ph.D. is Honorary Professor of Psychology at the University of British Columbia, 2136 West Mall, Vancouver, B.C., Canada, V6T 1Z4, (604) 822-9078. E-mail: acameron@psych.ubc.ca 
International Journal of Child, Youth and Family Studies (2012) 2 \& 3: 284-299

We open up this dialogue by asking the following questions:

- Can the current conceptual tools and language of child and youth care critically engage with the multiple and competing demands that practitioners, policy-makers, and researchers encounter in their everyday work?

- How can we capture lived childhoods without decontextualizing their meaning and yet still provide the information that is needed by policy-makers and practitioners?

We ask these questions because we believe that they are pressing questions for the contemporary researcher and that we cannot continue to investigate the field of child and youth care through disciplinary silos. There is no single lens through which one can begin to deconstruct the lived experience of children and youth. To understand their lived experiences we - as researchers and practitioners - must enter into the complex and dynamic bio-ecological system from which they - the children and youth - construct meaning. We need to do this because who we are grows from a complex and dynamic interaction between our biology and our environment in the broadest of terms.

Lee (2010) likens this dynamic interchange to a braid where the strands of human dispositions, physiological systems, environmental stimuli, and cultural practices cross over and interact with each other to shape who we become. She asks, “ . . . if adaptation and plasticity are characteristics of brain functioning ... why are we not doing more to understand the conditions of such adaptation and plasticity particularly with regard to those who face the greatest threats or obstacles ...?” (p. 647). Consequently, we argue that we need to use contemporary theories and methodologies of research that enable us to investigate the multiple pathways in which adaptation can occur. As researchers we need to become comfortable with methodologies that have the capacity to investigate systems and the transactional change that occurs between the interdependent parts. We need to become comfortable sitting at and even in the intersection of change. By considering only individual isolated parts of a system, our understanding of the adaptation process is limited and should not be used to drive either policy or practice. We believe that to inform and guide the policy and the practices of child and youth care counsellors, there is a need to recruit researchers that can cross multiple contexts and who are comfortable and competent with using a variety of research tools that have the capacity to investigate change transactionally, at the intersection of change across time.

In order to develop policy that leads to practice there is a need to understand the richness of ecology that shapes the lives of the children and youth, first via interaction and then transaction. Therefore, the focus of this paper will be to engage this discussion. Our intention here is to describe initially the bio-socio-ecological approaches of Bronfenbrenner (1994), Bronfenbrenner and Ceci (1994), Lee (2010), and Sameroff (2009a, 2009b, 2010), as we believe that their models provide a more holistic picture of the lived experience of childhood as they move between multiple contexts and have the capacity to direct child and youth care research. However, despite the many citations to this work within the human services field, there has been very little empirical research that focuses on specifically addressing the reciprocal effects between the child and the lived context (Sameroff, 2009a, 2009b). Then via a range of tools that 
International Journal of Child, Youth and Family Studies (2012) 2 \& 3: 284-299

have been utilized when investigating the resilience processes of early thriving (Cameron, 2009; Cameron, Tapanya, \& Gillen, 2006; Cameron, Ungar, \& Liebenberg, 2007; Gillen \& Cameron, 2010), we will provide a number of vignettes that will illustrate what can be learned about the complex lives of children and youth, when multiple methodologies that have the capacity to negotiate the rich ecology of childhood are used.

\section{Interactions and Transactions}

At this point one may ask, "How do interactions differ from transactions?” Sameroff (2009b) provides us with operational definitions of both of these terms. He defines interactions as “...dependencies in which the activity of one element is correlated with another" (p. 24) and provides the example of a smile being reciprocated with a smile as explanation. A transaction is identified as occurring “.... when the activity of one element changes the usual activity of another, either quantitatively by increasing or decreasing the usual level of response or qualitatively by eliciting or initiating a new response” (p. 24). He provides the example of when a smile is reciprocated with a frown, which may elicit confusion, negativity, or even increase anxious positivity as an explanation of the difference.

\section{Methodological Frameworks}

The bio-socio-ecological approaches of Bronfenbrenner (1994), Bronfenbrenner and Ceci (1994), and Sameroff (2009a, 2009b, 2010) hold a great deal of promise for guiding researchers who are interested in using new and existing models of research to capture and contextualize the complexity of the environment in which children and youth find themselves. These models of development direct our attention to the multiple pathways in which development can occur, and that we, as researchers, need to consider if our ways of working with children and youth are to be both meaningful and beneficial.

In their bio-ecological approach, Bronfenbrenner and Ceci (1994) describe developmental change as an interaction between multiple contexts and the child, and argue that to understand human development one must understand the entire ecological system in which the child grows. They draw to our attention to the importance of proximal processes in change and inform us that change occurs through the joint function of proximal process (i.e., the form, power, content, and direction of those processes) and the characteristics of the environment. Proximal processes are identified as mechanisms through which genetic potentials for effective psychological functioning are actualized. Bronfenbrenner and Ceci explain that proximal processes are involved with such functions as: differentiating perception and response, directing and controlling behaviour, coping successfully under stress, acquiring skills, and establishing relationships. They stress that underlying this model is a cardinal theoretical principle that states: "genetic material does not provide finished traits but rather interacts with environmental experience in determining developmental outcomes” (p. 571).

In this model the child is seen as developing within a system of bi-directional, interacting relationships. Bronfenbrenner (1994) identifies these bi-directional, interacting relationships as microsystems, mesosystems, exosystems, macrosystems, and chronosystems. A microsystem is created from a pattern of activities, social roles, and interpersonal relationships experienced by 
the developing child in a face-to-face or one-on-one experience. A mesosystem is comprised of the linkages between two or more systems such as home and school, whereas the exosystem is comprised of two or more systems that interact with each other but engages at least one system that the developing child does not inhabit. An example of this would be the parent's work environment. The child does not inhabit this environment but does experience its effects. The macrosystem represents the overarching beliefs of the culture or subculture the child lives within and involves such influences as beliefs, cultural customs, opportunities, and life course options. The chronosystem adds the historical change or time component within the individual and the environment (Bronfenbrenner, 1994).

Sameroff (2010) adds to this bio-psycho-social ecological approach with his unified theory of development. He identifies development as ongoing and transactional and states: "[t]he... development of any process in the individual is influenced by interplay with processes in the individual's context over time” (Sameroff, 2009a, p. 6). He describes a child as being a product of the continuous dynamic interactions between itself and the multiple social settings it needs to negotiate, and in doing so he draws our attention to the plasticity of both the environment and of the individual to engage in change. He adds that it is not just the child or the environment that changes in response to the interaction, but that both are changed as time moves forward due to the transactions that occur. To add to our increasing understanding of the complexity of this situation Sameroff (2009a, 2009b, 2010), like Bronfenbrenner (1994), addresses the fact that environments, like individuals, interact with each other and affect, and are affected by, each other.

Lee (2010) concurs with both Bronfenbrenner's (1994) and Sameroff's (2010) analysis of the systems of development being dynamically intertwined. She describes developmental change as involving human dispositions, physiological systems, cultural practices, and environmental stimuli and argues that these influences on development are so dynamically intertwined that it is only possible to understand human development through engaging with the multiple pathways that lead to adaptation.

As researchers we feel that Sameroff's (2010) unified theory holds a great deal of promise for directing the kind of research that needs to occur within the field of child and youth care. In support of Sameroff's model, a number of authors have described the need to move from a mechanistic world view of research to that of a systemic-organic world view (Cameron, 2009; Davison, 2006; Grajales \& Gonzales, 2008; Ramey \& Grubb, 2009), due to a growing need to understand any phenomenon within its context. There is a movement away from the acceptance of one simple reality or one simple answer that is independent from our perceptions, and movement toward an acceptance that each one of us experiences a different reality. Consequently, conducting research that fails to consider these differences or how they come about limits the findings to superficial factors, which involve only one or two dimensions of reality, and limits an understanding of the whole (Grajales \& Gonzalez, 2008). Ramey and Grubb (2009) argue that researchers need to spend time “...in a milieu of open critical dialogue” (p. 77) that would enable one to sift through the many different possibilities and forms of research to create new frameworks that do not privilege any one method or any one paradigm (Denzin \& Lincoln, 2005). This is good advice. Models must have the capacity to provide equal attention to the individual and to the environments in which individuals find themselves across 
International Journal of Child, Youth and Family Studies (2012) 2 \& 3: 284-299

time. Consequently, when completing research into the lived experience of children and youth, we need to move beyond the dominant, linear discourses that have traditionally been utilized within the field of child and youth care to those that have the capacity explore multilevel dynamic systems (Sameroff, 2010).

It is our belief that Sameroff's (2010) unified theory of development provides a place to have this critical dialogue, and the space to consider the types of methodologies that need to be used if one is to consider the transactional change between the child and his or her environment across time. There is a need for researchers to inhabit what Sameroff describes as "most known influences on the life trajectory" (p. 17) and then to spend time considering which models of investigation best serve the four models of change across time as they interact and then transact with each other. However, such a task is not to be taken lightly because, as Lee (2010) astutely reminds us, examining such interdependent concepts is conceptually and methodologically extremely difficult.

\section{Sameroff's Unified Theory}

In his unified theory Sameroff (2010) integrates four models of change and states that it is necessary to understand these models in order to understand human growth. Sameroff identifies these four models of change as: personal change, contextual, regulation, and representational. Personal change involves the change in an organism in the form of traits, growth, and development that occurs across the lifespan. Lee (2010) concurs with Sameroff about the importance of understanding this type of change due to our need to unpack the everchanging developmental complexities of the organism across time and then to plan for them. Contextual change refers to understanding the complexities of the environment that can constrain or promote development. Growing children find themselves in increasingly complex bioecological environments that they have to navigate. Consequently, the ability of the environment to support and then sustain the individual - directly or indirectly - dramatically affects the longterm outcomes and therefore needs to be understood. The regulation model describes the dynamic interface of self and the environment whereby early interactions during infancy are primarily biological but over time move to psychological (self) and social (other) regulation. Representational change explains the relationship between real world experiences and the development of thought in the form of the cognitive structures that stand for them. Real world experiences are encoded and stored and then used with increasing complexity as children age to provide an interpretive structure to their lived experience.

Sameroff in his 2010 discussions of this model clearly articulates the importance of considering these four models of change holistically, and transactionally. He states: "[c]ombining these four models offers a comprehensive view of the multiple parts, wholes and their connecting processes that comprise human development” (p. 12). He then continues to describe how these four models interact and transact across time to create one unified theory of development. In explaining his theory, Sameroff (2010) first starts with the components of the personal and contextual models, and then adds the regulation and the change components of the personal model to demonstrate the processes that need to be considered across time. He identifies self as being composed of a set of interacting psychological and biological processes that overlap the domains of emotional and cognitive development. In Figure 1 the psychological and 
biological processes are seen as a set of grey overlapping circles that compose the psychological self.

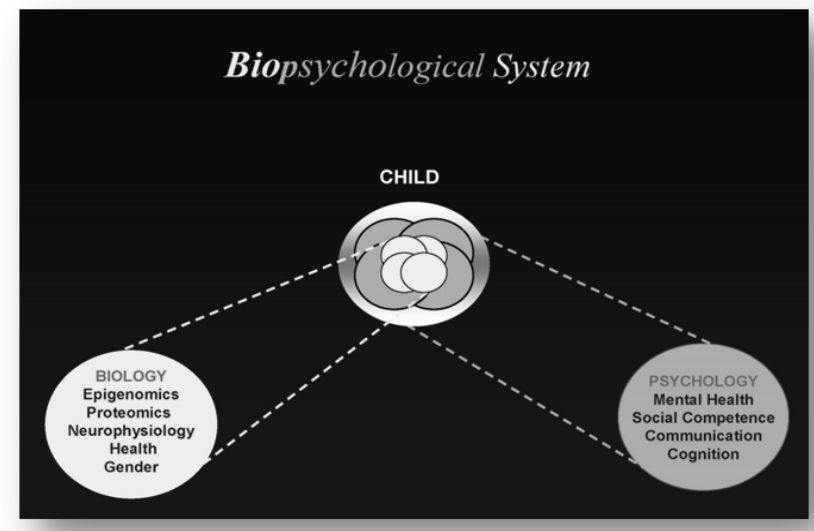

Figure 1. The Biopsychological System. Taken from "Predicting Developmental Outcomes: Is it the Child or the Environment” by A. Sameroff (2010), Oslo - November, 2010.

This biological self-system interacts with the ecology at large (Figure 2). This is the child's parents, siblings, and extended family and friends, the community at large, the school and their peers, and the geopolitical system of the time. The model now includes the biological and psychological components of self, interacting within the social ecology of the lived experience.

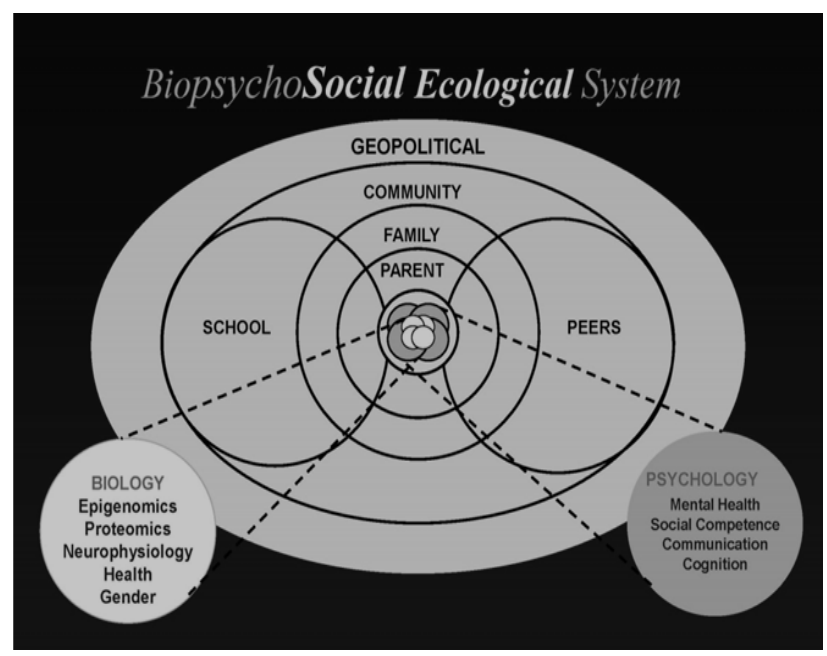

Figure 2. The Biopsychosocial Ecological System. Taken from "Predicting Developmental Outcomes: Is it the Child or the Environment” by A. Sameroff (2010), Oslo - November, 2010. 
Sameroff then adds the dimension of time (Figure 3) that he states can be used as a growth model in which bio-psychological aspects increase quantitatively across time without change in their interrelationship, or as a developmental model in which aspects of growth necessitate qualitative shifts in organization.

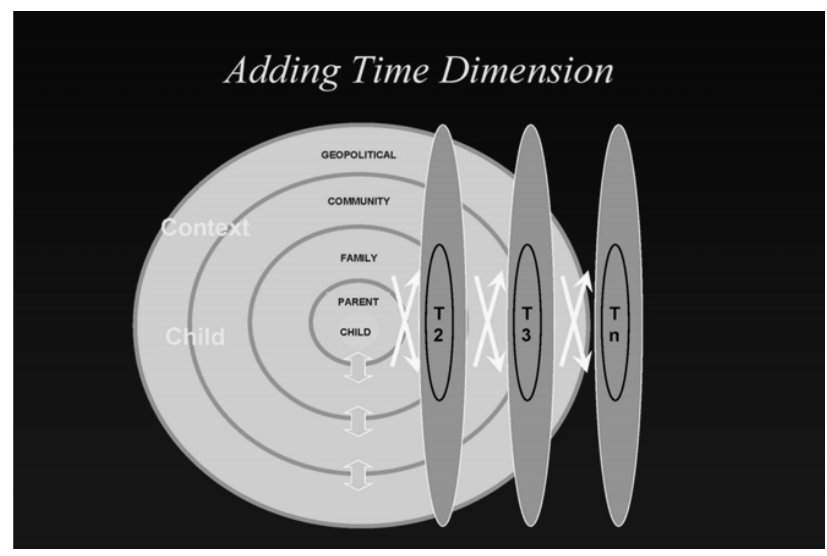

Figure 3. Adding the Time Dimension. Taken from "Predicting Developmental Outcomes: Is it the Child or the Environment” by A. Sameroff (2010), Oslo - November, 2010.

Figure 4 depicts the unified theory. Here Sameroff integrates the components of the biopsychological self, which includes the personal change and contextual and regulation models, within a social ecology across time. He excludes the representational model from the figure stating that it would make the model overly complex to add it, but reminds us that it is important to understand that it permeates every aspect of the model - that is, the interacting identities, attitudes, and beliefs of the child, the family, the culture, and the organizational structure of the social institutions.

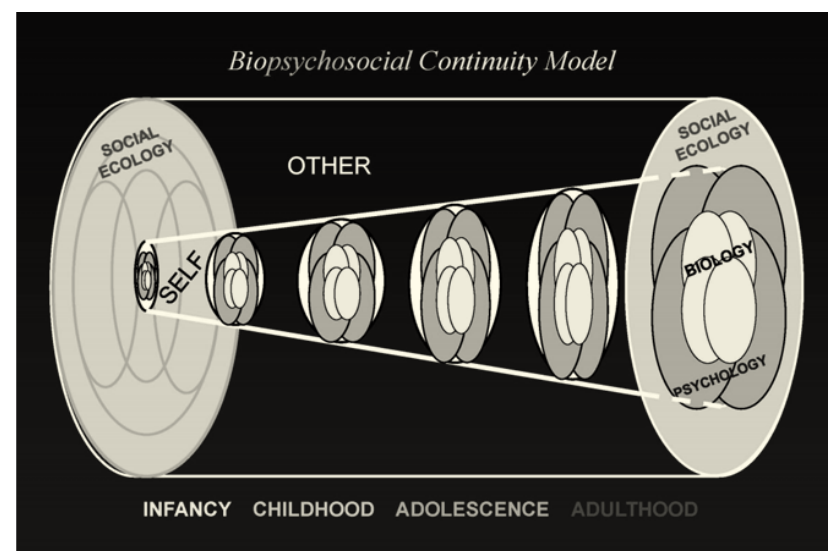

Figure 4. Biopsychosocial Continuity Model. Taken from "Predicting Developmental Outcomes: Is it the Child or the Environment” by A. Sameroff (2010), Oslo - November, 2010. 
Sameroff closes his discussion of the unified theory by emphasizing that it does not make any specific predictions about how development can occur but stresses that it explains what is necessary to consider if one wants to explain a development. This closing statement brings a focus to much we have learned about the children and youth we work with in the field of child and youth care as it has tremendous ramifications for research and practice in providing a rationale for why some of the work that we do simply does not make a difference whereas other work can and does.

Sameroff's unified theory provides an excellent model of how we should be conducting research and our practice within the field of child and youth care. In this model he provides clear examples of the component parts of the whole that interact and transact to create change across time. Therefore, as child and youth care researchers, we can use this model as an example of how to investigate the lived experience of children and youth and as a guideline for new and existing research tools that enable us to develop an understanding of how change occurs transactionally between and across domains within a given social ecology.

When thinking about the language of child and youth care and the importance of contextualized relationships in our work, utilizing a bio-psycho-socio-ecological approach such as the one suggested by Sameroff (2010) is most appropriate. This model fits with the philosophy of the field, and provides an opportunity to utilize a variety of investigative tools that work together transactionally. Using tools that can interact and transact will provide a picture of how longitudinal development is occurring within and across multiple domains in a complex ecology. These kinds of tools will enable us to ask the rich kinds of questions that only this type of framework can address.

\section{Unpacking our Current Research with Reference to the Unified Theory}

Sameroff (2010) describes the complexity of what he terms "untangling . . . social and biological processes [and the importance of not waiting] for a complete resolution of these pathways before [researchers] begin to attend to potential avenues of intervention” (p. 51). Therefore, to illustrate how we have begun to struggle with this issue, we offer five examples of methodologies drawn from our own research that we believe respond to Sameroff's call to engage in the kind of research that has the capacity to shed light on the multiple pathways through which adaptation can occur. The following examples utilize a variety of different research methods and tools to demonstrate how we have attempted to unpack and then reintegrate divergent methodologies that interact and transact with each other across time, thereby creating a more holistic picture of the lived experience of children and youth. All five examples involve the response of children and youth to stressful life experiences. Our first three examples focus on physiological processes in response to psychosocial stressors, showing how experimental manipulation in a laboratory setting can expose processes at the level of personal reactivity in the service of increasing psychological and social regulation of environmental challenges. Our final two examples are relatively naturalistic observational studies of the personal characteristics of thriving children and resilient youth in transition. We examined toddlers and teenagers in ecological context, in their homes and neighbourhoods respectively. 
International Journal of Child, Youth and Family Studies (2012) 2 \& 3: 284-299

We observed the models of change move from the biological to the psychosocial via the regulation of self and other. By having participants (parents of the toddlers and the youths themselves) reflect on their experiences, we were able to represent the interpretive structures of their lived experiences.

The first example is a project that investigated gender, anger, and attachment in adolescent stress responsivity. It involves controlled laboratory methods in order to identify causal factors in psychosocial stress reactivity. The typical biological response to a stressful situation is an increase in arousal mechanisms, indicating a state of heightened vigilance. This state can be identified by an increase in sympathetic arousal, such as elevated blood pressure and/or stress hormone levels (in particular, cortisol). However, research has not consistently demonstrated this pattern in adolescents' stress responding. Consequently, we were interested in determining why some studies show this change while others do not.

In order to dig a little deeper into this inconsistency in the literature, we conducted a study with a large sample of Canadian early adolescents and asked them to experience a stressful situation; participants performed an age-appropriate oral story-completion task before an audience of critical judges. The purpose of this study was to examine the role of individual differences in the teenagers' stress responses.

We found an expected bifurcation pattern when we examined our results. Half the teenagers showed an increase in arousal in response to the stressor whereas the other half actually decreased. This result held true for both boys and girls. In examining individual differences, we found that the girls who decreased in arousal were participants who self-reported low maternal attachment and high trait anger and who exhibited high teacher-reported aggression. In this study, these individual differences did not consistently predict whether boys would increase or decrease in arousal. However, this gender difference could be due to the type of anxiety-provoking stressor used.

Example two, following on from the above study, was designed to provide a more focused analysis of attachment and anger in youth. Additionally, this study included an investigation of coterminous anxiety, coping skills; and again, we elicited physiological stress responses. In this study, we used a different stress-inducing protocol and studied a different group of adolescents. This time, instead of requiring the youth to engage in public speaking, which can be anxiety provoking, we asked the adolescents to participate in an evaluated peer debate on a value-laden topic that we designed to produce frustration (a Frustration Social Stressor for Adolescents [FSS-A]).

We established a bifurcation pattern in response to this stressor as well. Half of the adolescents increased in rated physiological (cortisol) arousal whereas the other half decreased. The individual differences that predicted whether the adolescents' responses would increase or decrease was consistent with the previous study - adolescents reporting more positive maternal attachment and lower trait anger and rates of externalizing problems demonstrated greater cortisol reactivity in response to the FSS-A, while teenagers reporting high trait anger, poor maternal attachment, and whose teachers reported them as having high levels of externalizing behaviour showed a decrease in cortisol secretion. 
This time the individual differences that distinguished decreased from increased reactivity were consistent across genders. Although gender was related to different coping skills, it did not moderate cortisol reactivity. By combining the evidence from both studies, we found that low self-reported attachment and high trait anger is linked to a decremented biological response to stress.

When we consider both of these studies with respect to multiple pathways in which adolescent development can occur in the context of Sameroff's unified theory (2010), we can see data collected at the biological, and psychological level and at level of the bio-psychological self within a social ecology. At an individual biological level, we collected data on the adolescents' blood pressure and cortisol levels. Participants also completed a survey on their relationship with their parents and peers with specific interest in maternal attachment. The level of the teenagers' externalizing behaviour such as aggression was determined using ratings by the adolescents' teachers or parents. Additionally, by combining the results from the two studies one is able to start to develop an image of the interactions and the transactions that are taking place that associate with either an increased or decreased level of arousal, implicating the kinds of interventions that would be needed to ameliorate angry stress reactivity, namely working with establishing trust in developing attachment relationships, and thereby creating yet another beginning space for new investigations.

In a third, most recent, ongoing, study we are investigating younger children and their parents' stress responses to verbal deception. The stressor this time involves the need to make a moral choice, engaging the regulations of the children's and their parents' representations or interpretive structures in their physiological responsivity and, further, cultural differences between families are explored, thus engaging all four models of change in the unified theory. The child participants have to decide whether or not they should cover an apparent parental transgression.

The protocol of this study involves a (confederate) parent and their child, who are given a joint project to complete unsupervised. The parent is instructed to "find" an answer key and use it to complete the joint challenge, and then to "damage" accidentally some laboratory equipment when returning the key. Following this incident, the parent leaves the room and then the experimenter questions the child. The experimenter first queries the child about the high level of their performance on the joint task and then about the broken equipment. At this point the child has to decide either to cover for the parent or expose the transgressions. 
This was a significantly stressful experience for participants as indicated by Figures 5 and 6 , representing child and parents' normative cortisol increments.

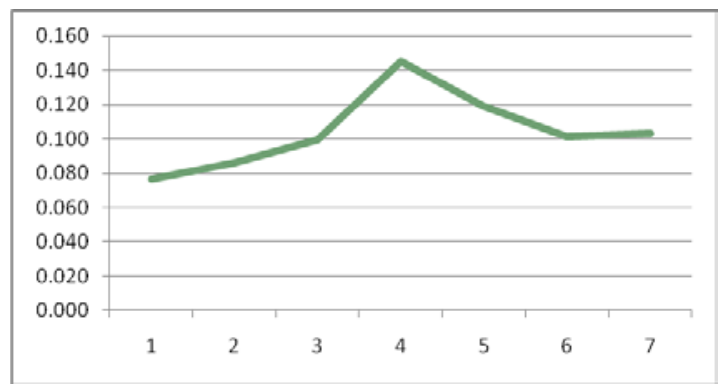

Figure 5. Cortisol responses of children who exposed parent and exhibited reactivity

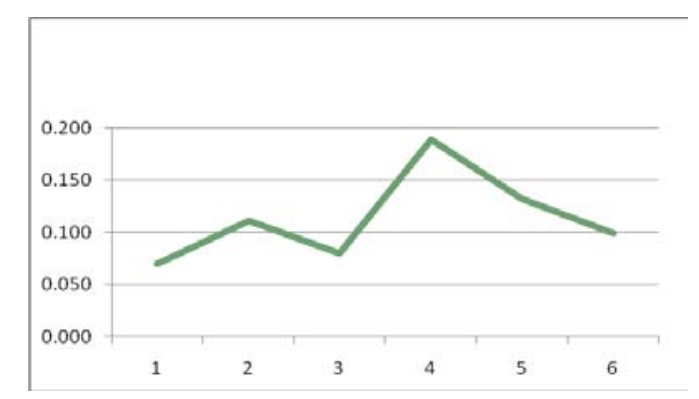

Figure 6. Cortisol responses of parents' normative reactivity to the stressful situation

Although we have worked with a relatively small number of participants to date, we have once again triggered the bifurcation pattern in stress response using this verbal deception stressor. Figures 7 and 8 depict the cortisol patterns of a parent and their related child whose levels decreased in response to the stressor.

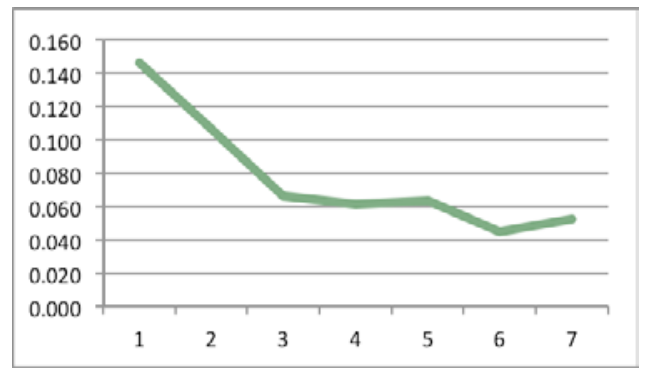

Figure 7. Parental decreased responsivity to the stressor. 


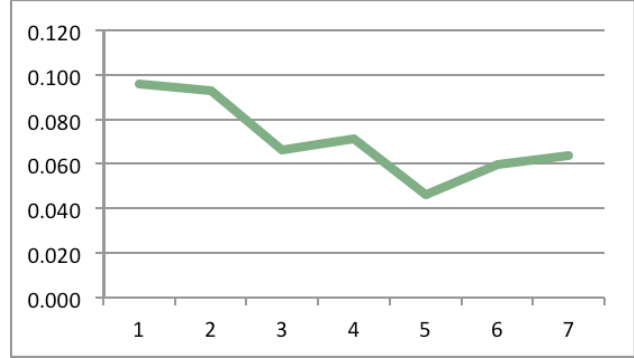

Figure 8. Paired child's decreased responsivity to the stressor.

As these are incomplete findings and data analyses are in progress, we are not yet able to determine the individual and contextual differences that predict which children and parents will increase or decrease in cortisol response to the stressor. We do however see consonance between family pairs in their reactivity, which is an interesting start in unravelling a relational picture. Nonetheless, by looking at different sources of data such as physiological responses, self-reports, parental and teachers' reports, we are able to expose this bifurcation pattern in at least three controlled situations that had not been captured by other procedures, highlighting both the importance of individual differences in the stress response and the advantages of pulling together different strands of youth experiences at the personal, contextual, regulatory, and representational levels of analysis.

New observational methodological approaches to child and youth transactions can be seen in two large international ecological studies using visual techniques to interrogate the daily experience of thriving toddlers and teenagers. They were cultural studies of everyday lives of children and adolescents in context. The major protocol for these studies was an innovative Day in the Life (DITL) filming of participants (Gillen et al., 2006). The procedure was initially developed to accommodate early childhood experiences in the home.

We filmed a full Day in the Life of 30-month-old girls in seven communities around the globe. Their parents were interviewed before we filmed their child and her family on a day (usually a weekend day or holiday) when parents were not working and the child was not in childcare out of the home. Parents were interviewed before the filming to ascertain their perspectives on childrearing and the characteristics of their child. After the filming, we made a compilation film of one half-hour out of the day, with approximately six clips of five minutes each that we thought represented some of the strengths revealed during the children's days. This composite film was shown to the parents who we encouraged to reflect further on their child, her strengths, and their socialization practices. We have published many studies of these toddlers' transactions in context, including their emotional security strivings, notational system developments (in drawing and joint book reading with parents), play, musicality, eating events, and humorous techniques for establishing themselves in familial connection. Some of these topics are represented in the Gillen and Cameron (2010) edited book.

A second application of this methodology to the habitus of resilient, migrant, adolescents around the globe involved some significant revisions (Cameron, 2011; Cameron, Theron, 
Liebenberg, \& Ungar, 2011). The new DITL visual approach was expanded to include a photo elicitation procedure and interviews with the participants themselves. Further, the teens orchestrated their own days to show what they thought were their strengths, knowing that they had been nominated by community youth advocates because they were seen as doing well under challenging, relocational adversity. Sixteen youths in eight locations (one boy, one girl in each) were filmed all in one day and again, a compilation film was made for them to reflect upon, as well as photographs they took of people, places, and things important to them.

Findings with the adolescents confirm that cultures and contexts matter. Similar themes play out very differently under varying circumstances and these variations are excellent signifiers that one-size-fits-all Western theoretical perspectives on resiliency processes underestimate the breadth and depth of developmental adaptation possibilities. Thriving reflects differential strengths at micro- to macro-systemic levels, from the personal through the contextual to the regulatory and representational systems. Traditional cultural practices in various contexts bring different strengths to migrant youths otherwise at risk for vulnerability (Theron et al., 2011). Some communities contribute promotive influences while others create barriers, depending upon circumstances. Schools play an especially large role in some communities whereas family strengths can scaffold autonomy in others. Emotional security is a hallmark of thriving but it has many forms (Cameron, Lau, \& Tapanya, 2009) and humour is a potent concomitant of enhanced socio-emotional functioning (E. Cameron, Fox, Anderson, \& A. Cameron, 2010). However, longitudinal work is necessary to reveal developmental patterns to these findings.

To conclude, we have offered five examples of our own work that we believe respond to Sameroff's (2010) call to engage in the kind of research that has the capacity to shed light on the multiple pathways through which adaptation can occur. These examples utilized research methods and tools aimed at demonstrating how we have attempted to unpack divergent methodologies that have the capacity to provide a more holistic picture of psychosocial stress as a lived experience of children and youth.

When considering Sameroff's (2010) model as a framework for practice and the importance of relationships and context to our work, utilizing a bio-psycho-socio-ecological approach is most appropriate. This model fits with the philosophy of the field, and provides an opportunity to utilize a variety of investigative tools that work together transactionally. Using tools that have the ability to interact and transact will provide a picture of how development is occurring within and across multiple domains in a complex ecology, longitudinally, and enable us to ask the rich kinds of questions that only this type of framework can address.

Sameroff's (2010) model as a framework for policy requires that we must learn to interpret both quantitative and qualitative data, insist upon using multimodal data in decisionmaking, and partner with children and youth enabling them to become their own spokespersons and advocates by using the data they provided from their lived experience.

Finally, as framework for research, Sameroff's (2010) unified theory provides a model of how we should be conducting research within the field of child and youth care. In this model he provides clear examples of the component parts of the whole that interact and transact to create change across time. However, it is important to recognize that although this theory has a strong 
dialectic, organismic orientation (Sameroff, 2009a, 2009b) we are not suggesting that one should only use a qualitative perspective, which is why we provided examples of how one can use both quantitative and qualitative methods to deepen understanding of psychosocial stress. In fact, many of the researchers that are calling for new methods and new ways of completing research are not suggesting that we should abandon quantitative methodologies, but that we should be moving away from the traditional binaries, and moving towards choosing eclectic methodologies that best suit the answer to the question (Davison, 2006; Yanchar, 2006a, 2006b). Yanchar (2006b) states: “. . . it is possible that some forms of quantitative research can fit theoretically within an interpretive framework and provide useful methodological resources for contextual interpretive enquiries. . . I . . argue, some quantitative strategies, when carefully interpreted and employed, can make integral contributions to the meaningful study of human action in context” (p. 212).

Yanchar makes this argument because he believes that we should investigate novel perspectives, methods, and strategies that may prove to be useful even though they may not follow traditional research methodologies. He emphasizes that there is a need to focus on theory construction, innovative methods, critical analysis, and problem solving rather than using "settled perspectives and formulaic methods" (p. 215). It is our belief that as researchers within the field of child and youth care, we should do this using Sameroff's unified theory as a model.

We began our discussions by asking if the current conceptual tools and language of child and youth care could critically engage with the multiple and competing demands of practitioners, policy-makers, and researchers and how it might be possible to capture the lived experience of childhood without decontextualizing its meaning and yet still providing the information that is needed. It is our belief that Sameroff's unified theory provides a theoretical model that will enable us to undertake this complex task. Additionally, we hope that by opening up the discussion on the unified theory as a framework for researchers within the field of child and youth care, others will join the dialogue, thereby deepening our understanding of how this model can be used as a framework for policy-makers, and practitioners as well. 
International Journal of Child, Youth and Family Studies (2012) 2 \& 3: 284-299

\section{References}

Bronfenbrenner, U. (1994). Ecological models of human development. In International encyclopedia of education (Vol. 3, 2nd ed.). Oxford: Elesevier. Reprinted in: M. Gavin \& M. Cole, (Eds.). (1993). Readings on the development of children (2nd ed., pp. 37-43). New York: Freeman.

Bronfenbrenner, U., \& Ceci, S. J. (1994). Nature-nurture reconceptualized in developmental perspective: A bioecological model. Psychological Review, 101(4), 568-586. doi:10.1037/0033-295X.101.4

Cameron, C. A. (2009). Constructing syntheses: Converging methods for investigating youth in diverse cultures. In L. Liebenberg \& M. Ungar (Eds.), Researching resilience (pp. 225244). Toronto: University of Toronto Press.

Cameron, C. A. (2011). “A Day in the Life”: A new methodology for investigating early childhood thriving and youth resilience around the globe. International Society for the Study of Behavioral Development, Bulletin, 1, Serial No. 59, 20-24.

Cameron, C. A, Lau, C., \& Tapanya, S. (2009). Passing it on during a Day in the Life of resilient adolescents in diverse communities around the globe. Child and Youth Care Forum, 38(5), 227-271.

Cameron, C. A., Tapanya, S., \& Gillen, J. (2006). Swings, hammocks, and rocking chairs as secure bases during A Day in the Life in diverse cultures. Child and Youth Care Forum, 35(3), 231-247.

Cameron, C. A., Theron, L., Ungar, M., \& Liebenberg, L. (2011). Adapting visual methodologies to identify youth protective processes in negotiating resilience across cultures and contexts. Australian Community Psychologist, 23(2), 68-84.

Cameron, C. A., Ungar, M., \& Liebenberg, L. (2007). Cross-cultural understandings of resilience: Roots for wings in the development of affective resources for resilience. In N. Carrey \& M. Ungar (Eds.), Resilience Special Issue. Child and Adolescent Psychiatric Clinics, 16(2), 285-301.

Cameron, E. L., Fox, J. D., Anderson, M. S., \& Cameron, C. A. (2010). Resilient youths use humor to enhance socio-emotional functioning during a Day in the Life. Journal of Adolescent Research, 25(5), 716-742.

Davison, K. G. (2006). Dialectical imagery in postmodern research. International Journal of Qualitative Studies in Education, 19(2), 133-146.

Denzin, N. K., \& Lincoln, Y. S. (Eds.). (2005). The Sage handbook of qualitative research (3rd ed.). Thousand Oaks, CA: Sage Publications. 
International Journal of Child, Youth and Family Studies (2012) 2 \& 3: 284-299

Gillen, J., \& Cameron, C. A. (Eds.). (2010). International perspectives on early childhood research: A Day in the Life. Houndmills, UK: Palgrave Macmillan.

Gillen, J., Cameron, C. A., Tapanya, S., Pinto, G., Hancock, R., Young, S., et al. (2006). “A Day in the Life": Advancing a methodology for the cultural study of development and learning in early childhood. Early Child Development and Care, 177(2), 207-218.

Grajales, T. E., \& Gonzales, S. (2008). Theory development: Towards a new concept of research. Journal of Research on Christian Education, 17(2), 153-172.

Lee, C. D. (2010). Soaring above the clouds, delving the ocean's depths: Understanding the ecologies of human learning and the challenge for educational science. Educational Research, 39(9), 643-655.

Ramey, H. J., \& Grubb, S. (2009). Modern, postmodernism and (evidence-based) practice. Contemporary Family Therapy, 31, 75-86.

Sameroff, A. (2009a). Designs for transactional research. In A. Sameroff (Ed.), The transactional model of development: How children and contexts shape each other (pp. 321). Washington, DC: American Psychological Association.

Sameroff, A. (2009b). The transactional model. In A. Sameroff (Ed.), The transactional model of development: How children and contexts shape each other (pp. 23-32). Washington, DC: American Psychological Association.

Sameroff, A. (2010). A unified theory of development: A dialectic integration of nature and nurture. Child Development, 81(1), 6-22.

Theron, L., Cameron, C. A., Didkowsky, N., Lau, C., Liebenberg, L., \& Ungar, M. (2011). A 'day in the life' of four resilient youths: Cultural roots of resilience. Youth and Society, 43(3), 799-881.

Yanchar, S. C. (2006a). On the possibility of contextual-quantitative inquiry. New Ideas in Psychology, 24(3), 212-228.

Yanchar, S. C. (2006b). Beyond the qualitative-quantitative divide: How to proceed? New Ideas in Psychology, 24(3), 275-281. 